Pacific Journal of Mathematic 


\title{
REALIZING TRANSFER MAPS FOR RAMIFIED COVERINGS
}

\author{
RALPH L. COHEN
}

\begin{abstract}
In this paper we study transfer maps for a general class of ramified covering spaces. This class includes finite covering spaces, as well as more general orbit maps $Y / H \rightarrow Y / G$, where $H$ is a subgroup of a finite group $G$, and where $Y$ is any $G$-space. This class also includes classical branched coverings of manifolds. The goal of this paper is to identify and to analyze the obstructions to realizing such a homology transfer homomorphism by a stable map between spaces.
\end{abstract}

The class of ramified coverings that we study was defined by $\mathrm{L}$. Smith. By the use of symmetric product spaces, Smith gave an elegant, combinatorial description of a transfer homomorphism

$$
\tau_{*}: H_{*}(X ; Z) \rightarrow H_{*}(\tilde{X} ; Z)
$$

for any $d$-fold ramified covering $\pi: \tilde{X} \rightarrow X$. In particular this homomorphism has the usual property that the composition

$$
\pi_{*} \tau_{*} ; H_{*}(X ; Z) \rightarrow H_{0}(X ; Z)
$$

is multiplication by $d$.

In the case of a regular covering map, or equivalently the orbit map of a free group action $X / H \rightarrow X / G$, it is well known that there exists a stable map (i.e. a map of suspension spectra)

$$
\tau: \Sigma^{\infty} X / G \rightarrow \Sigma^{\infty} X / H
$$

that realizes $\pi_{*}: H_{*}(X / G) \rightarrow H_{*}(X / H)$. These maps were discovered independently by Roush [8] and by Kahn and Priddy [4], and they have had several important applications in stable homotopy theory. In particular this was one of the main tools used in Kahn and Priddy's proof of their famous theorem that any stable map $\lambda: R P^{\infty} \rightarrow S^{0}$ that is nonzero on the first stable homotopy group, $\pi_{1}^{s}\left(_{-}\right)$, in fact induces a split surjection in 2-local homotopy groups in positive dimensions [4].

This naturally leads us to the following question. When does there exist stable realizations $\tau: \Sigma^{\infty} X \rightarrow \Sigma^{\infty} \tilde{X}$ of the transfer homomorphism of more general ramified covers $\pi: \tilde{X} \rightarrow X$ ? In his thesis [5], R. Kubelka showed that in general the induced transfer in $\bmod p$ cohomology, $\tau^{*}$ : 
$H^{*}(\tilde{X} ; \mathbf{Z} / p) \rightarrow H^{*}(X ; \mathbf{Z} / p)$ does not commute with the action of the Steenrod algebra, so such realizations do not in general exist.

In ths note we address the following local version of this question. If $n$ is an integer let $X[1 / n]$ denote the localizations of $X$ obtained by inverting all primes dividing $n$. So in particular $H_{*}(X[1 / n], \mathbf{Z}) \cong$ $H_{*}(X ; \mathbf{Z}) \otimes \mathbf{Z}[1 / n]$. In this paper we use Smith's homology transfer to give easy proofs of the following results, the first of which is a well known folk theorem.

THEOREM 1. Let $Y$ be an arbitrary $G$-space where $G$ is a finite group of order $n$. Let $\pi: Y \rightarrow Y / G$ be the orbit map. Then there exists a stable map of local spectra

$$
\tau: \Sigma^{\infty} Y / G\left[\frac{1}{n}\right] \rightarrow \Sigma^{\infty} Y\left[\frac{1}{n}\right]
$$

that realizes the local transfer homomorphism

$$
\tau_{*} \otimes 1: H_{*}(Y / G ; \mathbf{Z}) \otimes \mathbf{Z}\left[\frac{1}{n}\right] \rightarrow H_{*}(Y ; \mathbf{Z}) \otimes \mathbf{Z}\left[\frac{1}{n}\right] .
$$

THEOREM 2. Let $\pi: \tilde{X} \rightarrow X$ be any $d$-fold ramified cover in the sense of Smith [9]. Then there exists a stable map

$$
\tau: \Sigma^{\infty} X\left[\frac{1}{d !}\right] \rightarrow \Sigma^{\infty} \tilde{X}\left[\frac{1}{d !}\right]
$$

that realizes $\tau_{*} \otimes 1: H_{*}(X ; \mathbf{Z}) \otimes \mathbf{Z}[1 / d !] \rightarrow H_{*}(\tilde{X} ; \mathbf{Z}) \otimes \mathbf{Z}[1 / d !]$.

We will also observe that Theorem 2 is the best possible result in the most general setting. In particular we will exhibit an explicit 3-fold ramified cover $\pi: \tilde{X} \rightarrow X$ with the property that if $p$ is either 2 or 3 the induced cohomology transfer $\tau^{*}: H^{*}(\tilde{X} ; \mathbf{Z} / p) \rightarrow H^{*}(X: \mathbf{Z} / p)$ does not commute with the action of the Steenrod algebra. Thus both the primes 2 and 3 must be inverted before the transfer homomorphism can be realized by a stable map. We will also see that this phenomenon is general. That is, for every $d$ there exists a $d$-fold ramified cover $\pi: \tilde{X}_{d} \rightarrow X_{d}$ that has the property that for every prime $p \leq d$ the induced cohomology transfer $\tau^{*}$ : $H^{*}(\tilde{X} ; \mathbf{Z} / p) \rightarrow H^{*}(X ; \mathbf{Z} / p)$ does not respect the action of the Steenrod algebra.

The organization of this paper is as follows. In $\S 1$ we recall the Kahn-Priddy stable transfer map for free group actions, Smith's definition of general ramified coverings, and then describe the obstruction to obtaining stable transfer maps in the general setting. In $\S 2$ we prove Theorems 1 and 2 by showing that this obstruction vanishes after inverting the requisite set of primes. 
The author is grateful to G. Brumfiel for originally suggesting this problem to him and for several helpful conversations. He is also grateful to L. Smith for helpful correspondence. The author points out that Smith was aware of Theorem 2 and has an alternate proof.

1. The obstruction of realizing the transfer homomorphism. We begin by recalling the Kahn-Priddy stable transfer maps for free group actions [4].

Let $X$ be a space of the homotopy type of a C. W. complex and suppose it is acted upon freely on the right by a finite group $G$. Let $H<G$ be a subgroup of index $n$, and consider the $n$-fold covering

$$
p: X / H \rightarrow X / G
$$

given by the obvious projection of orbit spaces. By the left action of $G$ on the set of left cosets $G / H$ we get a representation

$$
\rho: G \rightarrow \Sigma_{n}
$$

where $\Sigma_{n}$ is the symmetric group on $n$-letters.

Let $E G$ and $E \Sigma_{n}$ denote contractible spaces that are acted upon freely on the left by $G$ and $\Sigma_{n}$ respectively. The representation induces an equivariant map $\rho_{*}: E G \rightarrow E \Sigma_{n}$. Kahn and Priddy define a map

$$
\Phi: X \times{ }_{G} E G \rightarrow X / H^{n} \times{ }_{\Sigma_{n}} E \Sigma_{n}
$$

by the formula $\Phi(x, w)=\left(\overline{x g_{1}}, \ldots, \overline{x g_{n}} ; \rho_{*}(w)\right)$, where $\left\{g_{1}, \ldots, g_{n}\right\} \in G$ represent the $n$ left cosets in $G / H$ and $\overline{x g_{i}}$ denotes the orbit of $x g_{i}$ in $X / H$. $G$ acts on the left of $x$ by $g x=x g^{-1}$, and $\Sigma_{n}$ acts on the left of $X / H^{n}$ by permuting coordinates.

Now recall from [3] that for any space $Y$ there are Dyer-Lashof maps $\alpha_{n}: Y^{n} \times_{\Sigma_{n}} E \Sigma_{n} \rightarrow \Omega^{\infty} \Sigma^{\infty} Y$. Letting $Y$ be $X / H$, define a stable map $T$ : $\Sigma^{\infty}\left(X \times{ }_{G} E G\right) \rightarrow \Sigma^{\infty} X / H$ to be the adjoint of the composition (which, by abuse of notation, we also call $T$ )

$$
T=\alpha_{n} \circ \Phi: X \times{ }_{G} E G \rightarrow X / H^{n} \times_{\Sigma_{n}} E \Sigma_{n} \rightarrow \Omega^{\infty} \Sigma^{\infty}(X / H) .
$$

Now since the action of $G$ on $X$ is free, the projection map $X \times{ }_{G} E G$ $\rightarrow X / G$ is a homotopy equivalence, so $T$ can be viewed as a stable map

$$
T: \Sigma^{\infty} X / G \rightarrow \Sigma^{\infty} X / H \text {. }
$$

In [4] Kahn and Priddy showed that $T_{*}: H_{*}(X / G) \rightarrow H_{*}(X / H)$ is the usual transfer homomorphism for covers as defined, for example in [10].

As we shall see, if $\pi: \tilde{X} \rightarrow X$ is a general ramified cover, the obstruction to obtaining a stable transfer map $T: \Sigma^{\infty} X \rightarrow \Sigma^{\infty} \tilde{X}$ is measured by the inability in general to describe $X$ in the form $\tilde{X} \times_{G} E G$. In 
order to make this precise, we now recall Smith's definition of a ramified covering and its corresponding transfer homomorphism [9].

Roughly speaking, a ramified covering is a surjective, finite to one map $\pi: \tilde{X} \rightarrow X$ together with a notion of multiplicity, so that if at each point $x \in X$ one writes down the unordered list of preimages $\left\{\pi^{-1}(x)\right\}$ according to their multiplicity, then this list varies continuously with $x \in X$. Here is how Smith makes this idea precise.

For every positive integer $d \in \mathbf{Z}^{+}$, let $\operatorname{SP}^{d}(Y)$ be the $d$-fold symmetric product $\operatorname{SP}^{d}(Y)=Y^{d} / \Sigma_{d}$, where again $\Sigma_{d}$ acts on $Y^{d}$ by permuting coordinates.

Definition 1.1 [9]. A surjective, finite to one map $\pi: \tilde{X} \rightarrow X$ is called a $d$-fold ramified covering iff there is a map

$$
\mu: X \rightarrow \mathbf{Z}^{+}
$$

called the multiplicity map such that

(1) For all $x \in X, \sum_{\tilde{x} \in \pi^{-1}(x)} \mu(\tilde{x})=d$,

(2) The function $\tau_{\pi}: X \rightarrow S P^{d}(\tilde{X})$ defined by sending $x$ into $\pi^{-1}(x)$, where each $\tilde{X} \in \pi^{-1}(x)$ occurs $\mu(x)$ times, is continuous.

As observed by Smith, this definition together with the Dold-Thom theorem [2] allows for an elegant description of the transfer homomorphism $\pi_{*}: H_{*}(X) \rightarrow H_{*}(\tilde{X})$ in the following manner.

For every integer $d$, one can include $Y^{d}$ into $Y^{d+1}$ as the subspace of $(d+1)$ - tuples whose last coordinate is a given fixed basepoint $y_{0} \in Y$. This respects the symmetric group actions, and so defines inclusions $\operatorname{SP}^{d}(Y) \rightarrow \operatorname{SP}^{d+1}(Y)$. Let

$$
\operatorname{SP}^{\infty}(Y)=\lim _{\vec{d}} \operatorname{SP}^{d}(Y) .
$$

THEOREM 1.2 (Dold and Thom [2]). $\mathrm{SP}^{\infty}(Y)$ is homotopy equivalent to the infinite loop space

$$
\lim _{\rightarrow} \Omega^{n}(K(\mathbf{Z}, n) \wedge Y)
$$

where $K(\mathbf{Z} ; n)$ is an Eilenberg-MacLane space of type $(\mathbf{Z}, n)$. Thus $\pi_{*} \operatorname{SP}^{\infty}(Y) \cong \tilde{H}_{*}(Y ; \mathbf{Z})$.

Now let $\pi: \quad \tilde{X} \rightarrow X$ be a $d$-fold ramified covering and consider the composition

$$
\tau: X \underset{\tau_{\pi}}{\rightarrow} \operatorname{SP}^{d}(\tilde{X}) \rightarrow \operatorname{SP}^{\infty}(\tilde{X})
$$


$\tau$ extends to a map, which by abuse we also call $\tau: \operatorname{SP}^{\infty} X \rightarrow \operatorname{SP}^{\infty} \tilde{X}$ defined by the maps

$$
\left(\tau_{\pi}\right)^{k}: \operatorname{SP}^{k}(X) \rightarrow \operatorname{SP}^{k d}(\tilde{X}) .
$$

Passing to a homotopy group, we get a transfer homomorphism

$$
\tau_{*}: \tilde{H}_{*}(X) \cong \pi_{*} \operatorname{SP}^{\infty}(X) \rightarrow \pi_{*} \operatorname{SP}^{\infty}(\tilde{X}) \cong \tilde{H}_{*}(\tilde{X}) \text {. }
$$

This homomorphism was studied in detail in [9] and was shown to have the usual properties of transfer homomorphisms. For example the following are easy corollaries of the definition (see [9]).

Proposition 1.3. Let $G$ be a finite group that acts on a space $Y$ with orbit map $\pi: Y \rightarrow Y / G$, structured as a $d=|G|$-fold ramified cover. Then the composition $\tau_{*} \circ \pi_{*}: \tilde{H}_{*} Y \rightarrow \tilde{H}_{*} Y$ is given by the formula

$$
\tau_{*} \pi_{*}(u)=\sum_{g \in G} g_{*}(u) \text {. }
$$

Proposition 1.4. Let $\pi: \tilde{X} \rightarrow X$ be a d-fold ramified cover. Then the composite homomorphism

$$
\pi_{*} \circ \tau_{*}: \tilde{H}_{*}(X) \rightarrow \tilde{H}_{*}(X)
$$

is multiplication by $d$.

REMARK. 1.4 is immediate after observing that the composite

$$
X \underset{\tau_{\pi}}{\rightarrow} \operatorname{SP}^{d}(\tilde{X}) \underset{\pi_{*}}{\rightarrow} \operatorname{SP}^{d}(X)
$$

is given by the $d$-fold diagonal map.

Now if $Y$ is any space consider the projection $h_{n}: Y^{n} \times_{\Sigma_{n}} E \Sigma_{n} \rightarrow$ $Y^{n} / \Sigma_{n}=\operatorname{SP}^{n}(Y)$. Observe that if $p: X / H \rightarrow X / G$ is the projection of orbit spaces coming from a $G$-action on $X$, then the following diagram commutes:

$$
\begin{array}{ccc}
X \times{ }_{G} E G & \stackrel{\Phi}{\rightarrow} & X / H^{n} \times_{\Sigma_{n}} E \Sigma_{n} \\
\downarrow f & & \downarrow h_{n} \\
X / G & \rightarrow & \operatorname{SP}^{n}(X / H)
\end{array}
$$

where $f$ is the projection map and $n$ is the index of $H$ in $G$. Thus if the action is free and hence $f$ is a homotopy equivalence, the Kahn-Priddy map $\Phi: X / G \rightarrow X / H^{n} \times_{\Sigma_{n}} E \Sigma_{n}$ that was used to define the stable transfer map, homotopy lifts the Smith map $\tau_{p}: X / G \rightarrow \operatorname{SP}^{n}(X / H)$ that was used to define the homology transfer homomorphism. 
For a general ramified cover $\pi: \tilde{X} \rightarrow X$, the obstruction to the existence of a such a homotopy lifting of $\tau_{\pi}$ will measure our obstruction to the existence of a stable transfer map $\tau: \Sigma^{\infty} X \rightarrow \Sigma^{\infty} \tilde{X}$. We end this section by identifying this obstruction more precisely.

Let $\pi: \quad \tilde{X} \rightarrow X$ be a $d$-fold ramified cover and let $C(h)$ be the homotopy cofiber (i.e. mapping cover) of $h_{d}: \tilde{X}^{d} \times_{\Sigma_{d}} E \Sigma_{d} \rightarrow \operatorname{SP}^{d}(\tilde{X})$. For $n$ a positive integer let $\{X, C(h)[1 / n]\}$ be the abelian group of stable maps (i.e. maps of suspension spectra) from $X$ to the localization of $C(h)$. We then define a class

$$
\boldsymbol{\theta}_{\bar{\pi}}\left[\frac{1}{n}\right] \in\left\{X, C(h)\left[\frac{1}{n}\right]\right\}
$$

to be represented by the localization of the composition

$$
X \underset{\tau_{\pi}}{\rightarrow} \operatorname{SP}^{d}(\tilde{X}) \underset{j}{\rightarrow} C(h)
$$

where $j$ is the inclusion of $\operatorname{SP}^{d}(\tilde{X})$ into the mapping cone of $h_{d}$.

Proposition 1.5. If $\theta_{\pi}[1 / n] \in\{X, C(h)[1 / n]\}$ is zero then there exists a map of localized suspension spectra

$$
T: \Sigma^{\infty} X\left[\frac{1}{n}\right] \rightarrow \Sigma^{\infty} \tilde{X}\left[\frac{1}{n}\right]
$$

so that in homology,

$$
T_{*}=\tau_{*} \otimes 1: \tilde{H}_{*}(X ; \mathbf{Z}) \otimes \mathbf{Z}\left[\frac{1}{n}\right] \rightarrow \tilde{H}_{*}(\tilde{X}, \mathbf{Z}) \otimes \mathbf{Z}\left[\frac{1}{n}\right] .
$$

Proof. By definition, it is immediate that $\theta_{\pi}[1 / n]$ is the obstruction to the existence of a stable homotopy lifting

$$
\tilde{T}: \Sigma^{\infty} X\left[\frac{1}{n}\right] \rightarrow \Sigma^{\infty}\left(\tilde{X}^{d} \times_{\Sigma_{d}} E \Sigma_{d}\right)\left[\frac{1}{n}\right]
$$

of the localization of $\tau: X \rightarrow \operatorname{SP}^{d}(\tilde{X})$. It is therefore sufficient to show how to construct the required map $T: \Sigma^{\infty} X[1 / n] \rightarrow \Sigma^{\infty} \tilde{X}[1 / n]$ out of such a lifting $\tilde{T}$.

For any space $Y$ let

$$
H: \Omega^{\infty} \Sigma^{\infty} Y \rightarrow \operatorname{SP}^{\infty}(Y) \simeq \lim _{\rightarrow} \Omega^{q}(K(\mathbf{Z}, q) \wedge Y)
$$

$q$

be the infinite loop map induced by the inclusions

$$
\Sigma^{q} Y=S^{q} \wedge \underset{i \wedge 1}{\rightarrow} K(\mathbf{Z}, q) \wedge Y
$$


where $i \in \pi_{q} K(\mathbf{Z}, q)=\mathbf{Z}$ is a generator. It is clear that $h_{*}: \pi_{*}\left(\Omega^{\infty} \Sigma^{\infty} Y\right)$ $\rightarrow \pi_{*}\left(\mathrm{SP}^{\infty}(Y)\right)$ is the stable Hurewicz homomorphism $h_{*}: \pi_{*}^{s}(Y) \rightarrow$ $\tilde{H}_{*}(Y)$. Moreover, it is also well known (see [6] for example) that the following diagram homotopy commutes:

$$
\begin{array}{ccc}
Y^{k} \times_{\Sigma_{k}} E \Sigma_{k} & \stackrel{\alpha_{k}}{\rightarrow} & \Omega^{\infty} \Sigma^{\infty} Y \\
\downarrow h_{k} & & \downarrow h \\
\operatorname{SP}^{k}(Y) & \rightarrow & \operatorname{SP}^{\infty}(Y)
\end{array}
$$

where $\alpha_{k}$ is the Dyer-Lashof map mentioned above.

Thus a lifting

$$
\tilde{T}: \Sigma^{\infty} X\left[\frac{1}{n}\right] \rightarrow \Sigma^{\infty}\left(\tilde{X}^{d} \times_{\Sigma_{d}} E \Sigma_{d}\right)\left[\frac{1}{n}\right]
$$

of the localization of $\tau: \Sigma^{\infty} X \rightarrow \Sigma^{\infty} \mathrm{SP}^{d} \tilde{X}$ allows us to define a stable map

$$
\bar{T}: \Sigma^{\infty} X\left[\frac{1}{n}\right] \underset{\tilde{T}}{\rightarrow} \Sigma^{\infty}\left(\tilde{X}^{d} \times_{\Sigma_{d}} E \Sigma_{d}\right)\left[\frac{1}{n}\right] \underset{\alpha_{d}}{\rightarrow} \Sigma^{\infty}\left(\Omega^{\infty} \Sigma^{\infty} \tilde{X}\right)\left[\frac{1}{n}\right]
$$

or equivalently, its adjoint

$$
\bar{T}: X\left[\frac{1}{n}\right] \rightarrow \Omega^{\infty} \Sigma^{\infty}\left(\Omega^{\infty} \Sigma^{\infty} \tilde{X}\right)\left[\frac{1}{n}\right],
$$

that homotopy lifts the localization of the composition

$$
\bar{\tau}: X \underset{\tau_{\pi}}{\rightarrow} \operatorname{SP}^{d}(\tilde{X}) \rightarrow \operatorname{SP}^{\infty}(\tilde{X}) \rightarrow \Omega^{\infty} \Sigma^{\infty}\left(\operatorname{SP}^{\infty}(\tilde{X})\right) .
$$

Now since $\Omega^{\infty} \Sigma^{\infty} \tilde{X}$ and $\operatorname{SP}^{\infty} \tilde{X}$ are infinite loop spaces and $h: \Omega^{\infty} \Sigma^{\infty} X \rightarrow$ $\operatorname{SP}^{\infty} X$ is an infinite loop map, there exist infinite loop retractions $a$ : $\Omega^{\infty} \Sigma^{\infty}\left(\Omega^{\infty} \Sigma^{\infty} \tilde{X}\right) \rightarrow \Omega^{\infty} \Sigma^{\infty} \tilde{X}$ and $b: \quad \Omega^{\infty} \Sigma^{\infty}\left(\operatorname{SP}^{\infty}(\tilde{X})\right) \rightarrow \operatorname{SP}^{\infty}(\tilde{X})$ that make the following diagram homotopy commute:

$$
\begin{array}{ccc}
\Omega^{\infty} \Sigma^{\infty}\left(\Omega^{\infty} \Sigma^{\infty} \tilde{X}\right) & \rightarrow & \Omega^{\infty} \Sigma^{\infty} \tilde{X} \\
\downarrow \Omega^{\infty} \Sigma^{\infty} h & & \downarrow h \\
\Omega^{\infty} \Sigma^{\infty}\left(\operatorname{SP}^{\infty}(\tilde{X})\right) & \stackrel{b}{\rightarrow} & \operatorname{SP}^{\infty}(\tilde{X}) .
\end{array}
$$

Thus the composition

$$
T: X\left[\frac{1}{n}\right] \underset{\bar{T}}{\rightarrow} \Omega^{\infty} \Sigma^{\infty}\left(\Omega^{\infty} \Sigma^{\infty} \tilde{X}\right)\left[\frac{1}{n}\right] \underset{a}{\rightarrow} \Omega^{\infty} \Sigma^{\infty} \tilde{X}\left[\frac{1}{n}\right]
$$

lifts the localization of $\tau: X \rightarrow \operatorname{SP}^{\infty} \tilde{X}$. By abuse of notation we let $T$ also represent the adjoint

$$
T: \Sigma^{\infty} X\left[\frac{1}{n}\right] \rightarrow \Sigma^{\infty} \tilde{X}\left[\frac{1}{n}\right]
$$


It is now clear that in homology $T$ induces

$$
\tau_{*} \otimes 1: \tilde{H}_{*}(X) \otimes \mathbf{Z}\left[\frac{1}{n}\right] \rightarrow \tilde{H}_{*}(\tilde{X}) \otimes \mathbf{Z}\left[\frac{1}{n}\right]
$$

and hence the proposition is proved.

2. Proofs of Theorems 1 and 2. We now use Proposition 1.5 to prove Theorems 1 and 2.

Proof of Theorem 1. As in the statement of the theorem we let $Y$ be any $G$-space, $n=$ order of $G$, and $\pi: Y \rightarrow Y / G$ the orbit map. Consider the following homotopy commutative diagram

$$
\begin{array}{ccc}
Y \times_{G} E G & \stackrel{\Phi}{\rightarrow} & Y^{n} \times_{\Sigma_{n}} E \Sigma_{n} \\
\downarrow f & & \downarrow h_{n} \\
Y / G & \rightarrow & \operatorname{SP}^{n}(Y) .
\end{array}
$$

Now it well known (see [1] for example) that the projection map $f$ induces an isomorphism in localized homology

$$
f_{*}: \tilde{H}_{*}\left(Y \times_{G} E G ; \mathbf{Z}\left[\frac{1}{n}\right]\right) \stackrel{\cong}{\rightarrow} \tilde{H}_{*}\left(Y / G ; \mathbf{Z}\left[\frac{1}{n}\right]\right)
$$

and hence after inverting $n=|G|, f$ becomes a stable homotopy equivalence. Let

$$
g: \Sigma^{\infty} Y / G\left[\frac{1}{n}\right] \rightarrow \Sigma^{\infty}\left(Y \times{ }_{G} E G\right)\left[\frac{1}{n}\right]
$$

be a stable homotopy inverse. Then by the commutativity of this diagram, the composition

$$
\Sigma^{\infty} Y / G\left[\frac{1}{n}\right] \underset{g}{\rightarrow} \Sigma^{\infty}\left(Y \times_{G} E G\right)\left[\frac{1}{n}\right] \underset{\Phi}{\rightarrow} \Sigma^{\infty}\left(Y^{n} \times_{\Sigma_{n}} E \Sigma_{n}\right)\left[\frac{1}{n}\right]
$$

is a stable homotopy lifting of the localization of $\tau: Y / G \rightarrow \operatorname{SP}^{\infty}(Y)$. Thus $\theta_{\pi}[1 / n] \in\{Y / G, C(h)[1 / n]\}$ is trivial. Hence Theorem 1 follows from 1.5.

Proof of Theorem 2. Let $\pi: \tilde{X} \rightarrow X$ be an arbitrary $d$-fold ramified covering. Since $d !=$ the order of $\Sigma_{d}$, then the map

$$
h_{d}: \tilde{X}^{d} \times_{\Sigma_{d}} E \Sigma_{d} \rightarrow \operatorname{SP}^{d}(\tilde{X})=\tilde{X}^{d} / \Sigma_{d}
$$

induces an isomorphism in localized homology,

$$
h_{d_{*}}: \tilde{H}_{*}\left(\tilde{X}^{d} \times_{\Sigma_{d}} E \Sigma_{d} ; \mathbf{Z}\left[\frac{1}{d !}\right]\right) \underset{\cong}{\rightarrow} \tilde{H}_{*}\left(\operatorname{SP}^{d}(\tilde{X}) ; \mathbf{Z}\left[\frac{1}{d !}\right]\right) .
$$


Thus after inverting $d !, h_{d}$ becomes a stable homotopy equivalence. This says that the localized mapping cone, $C(h)[1 / d !]$ is stably contractible. Thus $\theta_{\pi}[1 / d !] \in\{X, C(h)[1 / d !]\}$ is trivial, and so Theorem 2 follows from 1.5.

We end this paper by giving examples to show that Theorem 2 is the best possible result in the general ramified cover setting. We first exhibit a 3-fold ramified cover for which, because of Steenrod algebra considerations, it is necessary to invert both the primes 2 and 3 before one can stably realize the transfer homomorphism.

Let $\Sigma_{3}$ act on $S^{2} \times S^{2} \times S^{2}$ be permuting coordinates and let $\Sigma_{2} \subset \Sigma_{3}$ be the subgroup of elements that leave the first coordinate fixed. Consider the orbit space projection map

$$
\pi:\left(S^{2}\right)^{3} / \Sigma_{2} \rightarrow\left(S^{2}\right)^{3} / \Sigma_{3}=\operatorname{SP}^{3}\left(S^{2}\right) .
$$

$\pi$ is a 3-fold ramified cover.

Now $\left(S^{2}\right)^{3} / \Sigma_{2} \cong \operatorname{SP}^{2}\left(S^{2}\right) \times S^{2}$ so therefore

Moreover,

$$
\tilde{H}_{q}\left(\left(S^{2}\right)^{3} / \Sigma_{2} ; \mathbf{Z}\right)= \begin{cases}\mathbf{Z} \oplus \mathbf{Z} & \text { if } q=2 \text { or } 4 \\ \mathbf{Z} & \text { if } q=6 \\ 0 & \text { otherwise. }\end{cases}
$$

$$
\tilde{H}_{q}\left(\operatorname{SP}^{3}\left(S^{2}\right) ; \mathbf{Z}\right)= \begin{cases}\mathbf{Z}, & \text { if } q=2,4, \text { or } 6 \\ 0 & \text { otherwise. }\end{cases}
$$

Now let $p=2$ or 3 and $A$ the mod $p$ Steenrod algebra. The dual, $A_{*}=\operatorname{Hom}(A, \mathbf{Z} / 2)$ acts on $\bmod p$ homology. The following diagrams describe the $A_{*}$-module structures of the homology of these spaces. (The nodes represent generators.)

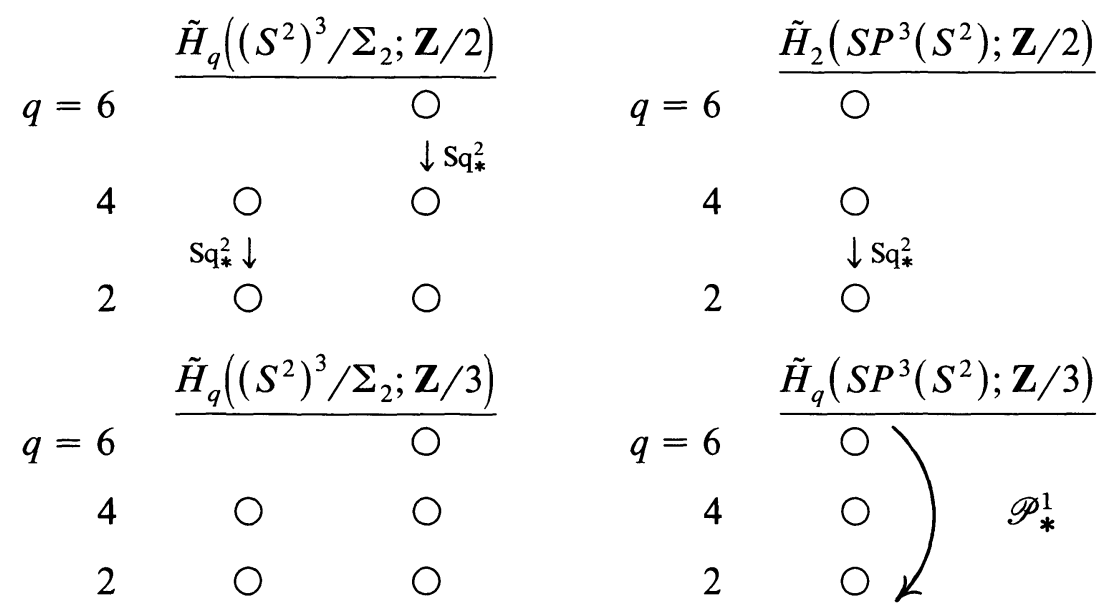

(trivial $A_{*}$-action) 
The reference for these homology structures is [7]. A quick examination of these diagrams yields that the transfer homomorphisms

$$
\tau_{*}: \tilde{H}_{*}\left(\operatorname{SP}^{3}\left(S^{2}\right) ; \mathbf{Z} / p\right) \rightarrow \tilde{H}_{*}\left(\left(S^{2}\right)^{3} / \Sigma_{2} ; \mathbf{Z} / p\right)
$$

do not preserve the $A_{*}$-structures. Thus $\tau_{*}$ cannot be realized by a stable map unless both the primes 2 and 3 are inverted.

We remark that more general examples of $d$-fold ramified covers that have Steenrod algebra obstructions to constructing stable transfer maps unless $d$ ! is inverted can be found in a similar manner. Namely, one studies ramified covers of the sort

$$
\pi: X^{d} / \Sigma_{d-1} \rightarrow X^{d} / \Sigma_{d}=\operatorname{SP}^{d}(X) .
$$

We leave the details to the reader.

\section{REFERENCES}

[1] G. E. Bredon, Introduction to Compact Transformation Groups, Academic Press, New York-London, 1972.

[2] A. Dold and R. Thom, Quasifaserungen und unendliche symmetrische produkte, Annals of Math., 67 91958), 239-281.

[3] E. Dyer and R. Lashof, Homology of iterated loop spaces, Amer. J. Math., 84 91962), 35-88.

[4] D. S. Kahn and S. B. Priddy, Applications of the transfer to stable homotopy theory, Bull. Amer. Math. Soc., 78 \#6 (1972), 981-987.

[5] R. Kubelka, Ph. D. Thesis, Stanford Univ., 1980.

[6] J. P. May, The Geometry of Iterated Loop Spaces, Springer Lecture Notes \#271, 1972.

[7] M. Nakaoka, Cohomology mod $p$ of symmetric products of spheres II, J. of Inst. of Polytechnics, Osaka City Univ., 10 \#2 series A (1959), 67-89.

[8] F. W. Roush, Transfer in generalized cohomology theories, $\mathrm{Ph}$. D. thesis, Princeton Univ., 1971.

[9] L. Smith, Transfer and ramified coverings, Math. Proc. Comb. Phil. Soc., 93 (1983), 485-493.

[10] N. E. Steenrod and D. B. A. Epstein, Cohomology Operations, Annals of Math. Studies \#50, 1962.

[11] R. E. Stong, Cobordism of branched covers, University of Virginia preprints (1981).

Received November 16, 1984. The author was partially supported by N. S. F. grant MCS82-03806 and by an A. P. Sloan Foundation fellowship.

PRINCETON UNIVERSITY

PRINCETON, NJ 08544

AND

STANFORD UNIVERSITY

STANFORD, CA 94305 


\section{PACIFIC JOURNAL OF MATHEMATICS EDITORS}

\author{
V. S. VARAdarajan (Managing Editor) \\ University of California \\ Los Angeles, CA 90024 \\ Hebert Clemens \\ University of Utah \\ Salt Lake City, UT 84112 \\ Charles R. DePrima \\ California Institute of Technology \\ Pasadena, CA 91125
}

R. FINN

Stanford University

Stanford, CA 94305

HeRManN FLASChKa

University of Arizona

Tucson, AZ 85721

RAMESH A. GANGOlli

University of Washington

Seattle, WA 98195

ROBION KIRBY

University of California

Berkeley, CA 94720

\author{
C. C. MOORE \\ University of California \\ Berkeley, CA 94720 \\ H. SAMELSON \\ Stanford University \\ Stanford, CA 94305 \\ HAROLD STARK \\ University of California, San Diego \\ La Jolla, CA 92093
}

\section{ASSOCIATE EDITORS}

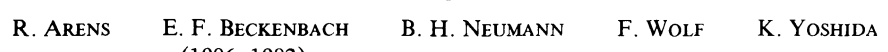

(1906-1982)

\section{SUPPORTING INSTITUTIONS}

\begin{abstract}
UNIVERSITY OF ARIZONA
UNIVERSITY OF BRITISH COLUMBIA

CALIFORNIA INSTITUTE OF TECHNOLOGY

UNIVERSITY OF CALIFORNIA

MONTANA STATE UNIVERSITY

UNIVERSITY OF NEVADA, RENO

NEW MEXICO STATE UNIVERSITY

OREGON STATE UNIVERSITY
\end{abstract}

\author{
UNIVERSITY OF OREGON \\ UNIVERSITY OF SOUTHERN CALIFORNIA \\ STANFORD UNIVERSITY \\ UNIVERSITY OF HAWAII \\ UNIVERSITY OF TOKYO \\ UNIVERSITY OF UTAH \\ WASHINGTON STATE UNIVERSITY \\ UNIVERSITY OF WASHINGTON
}

The Supporting Institutions listed above contribute to the cost of publication of this Journal, but they are not owners or publishers and have no responsibility for its content or policies.

Mathematical papers intended for publication in the Pacific Journal of Mathematics should be in typed form or offset-reproduced (not dittoed), double spaced with large margins. Please do not use built up fractions in the text of the manuscript. However, you may use them in the displayed equations. Underline Greek letters in red, German in green, and script in blue. The first paragraph must be capable of being used separately as a synopsis of the entire paper. In particular it should contain no bibliographic references. Please propose a heading for the odd numbered pages of less than 35 characters. Manuscripts, in triplicate, may be sent to any one of the editors. Please classify according to the scheme of Math. Reviews, Index to Vol. 39. Supply name and address of author to whom proofs should be sent. All other communications should be addressed to the managing editor, or Elaine Barth, University of California, Los Angeles, California 90024.

There are page-charges associated with articles appearing in the Pacific Journal of Mathematics. These charges are expected to be paid by the author's University, Government Agency or Company. If the author or authors do not have access to such Institutional support these charges are waived. Single authors will receive 50 free reprints; joint authors will receive a total of 100 free reprints. Additional copies may be obtained at cost in multiples of 50 .

The Pacific Journal of Mathematics is issued monthly as of January 1966. Regular subscription rate: $\$ 190.00$ a year (5 Vols., 10 issues). Special rate: $\$ 95.00$ a year to individual members of supporting institutions.

Subscriptions, orders for numbers issued in the last three calendar years, and changes of address should be sent to Pacific Journal of Mathematics, P.O. Box 969, Carmel Valley, CA 93924, U.S.A. Old back numbers obtainable from Kraus Periodicals Co., Route 100, Millwood, NY 10546.

The Pacific Journal of Mathematics at P.O. Box 969, Carmel Valley, CA 93924 (ISSN 0030-8730) publishes 5 volumes per year. Application to mail at Second-class postage rates is pending at Carmel Valley, California, and additional mailing offices. Postmaster: Send address changes to Pacific Journal of Mathematics, P.O. Box 969, Carmel Valley, CA 93924.

PUBLISHED BY PACIFIC JOURNAL OF MATHEMATICS, A NON-PROFIT CORPORATION

Copyright $\odot 1986$ by Pacific Journal of Mathematics 


\section{Pacific Journal of Mathematics}

\section{Vol. 122, No. 2 \\ February, 1986}

Gideon Amit and David Chillag, On a question of Feit concerning character values of finite solvable groups ......................257

Constantin Gelu Apostol and Frank Larkin Gilfeather, Isomorphisms modulo the compact operators of nest algebras ................263

Parviz Azimi and James Neil Hagler, Examples of hereditarily $l^{1}$ Banach

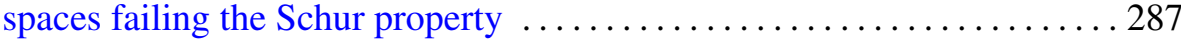

Brian Evan Blank, Boundary behavior of limits of discrete series representations of real rank one semisimple groups . . . . . . . . . . 299

Jeffrey Carroll, Some undecidability results for lattices in recursion theory

Gerald Howard Cliff and Alfred Rheinhold Weiss, Crossed product and

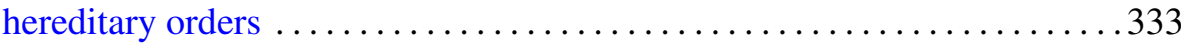

Ralph Cohen, Realizing transfer maps for ramified coverings . . . . . . . . 347

Ronald James Evans, Hermite character sums . .................. 357

C. L. Frenzen and Roderick Sue-Chuen Wong, Asymptotic expansions of the Lebesgue constants for Jacobi series . . . . . . . . . . . . . . . . 391

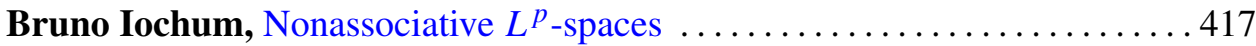

John McDonald, Unimodular approximation in function algebras ....... 435

John Robert Quine, Jr., Ramification and unintegrated value distribution . . 441

Marc Raphael, Commutants of quasisimilar subnormal operators ........ 449

Parameswaran Sankaran and Peter Zvengrowski, On stable

parallelizability of flag manifolds

Helga Schirmer, A relative Nielsen number

Barry Simon, Schrödinger semigroups on the scale of Sobolev spaces . . . . . 475

Viakalathur Shankar Sunder, Stochastic integration in Fock space

Jan de Vries, A note on the $G$-space version of Glicksberg's theorem 